\title{
g
}

\section{Precise Quantization of the Anomalous Hall Effect near Zero Magnetic Field}

\author{
A. J. Bestwick, ${ }^{1,2}$ E. J. Fox, ${ }^{1,2}$ Xufeng Kou, ${ }^{3}$ Lei Pan, ${ }^{3}$ Kang L. Wang, ${ }^{3}$ and D. Goldhaber-Gordon ${ }^{1,2, *}$ \\ ${ }^{1}$ Department of Physics, Stanford University, Stanford, California 94305, USA \\ ${ }^{2}$ Stanford Institute for Materials and Energy Sciences, SLAC National Accelerator Laboratory, \\ 2575 Sand Hill Road, Menlo Park, California 94025, USA \\ ${ }^{3}$ Department of Electrical Engineering, University of California, Los Angeles, California 90095, USA
}

(Received 16 January 2015; revised manuscript received 16 March 2015; published 4 May 2015)

\begin{abstract}
We report a nearly ideal quantum anomalous Hall effect in a three-dimensional topological insulator thin film with ferromagnetic doping. Near zero applied magnetic field we measure exact quantization in the Hall resistance to within a part per 10000 and a longitudinal resistivity under $1 \Omega$ per square, with chiral edge transport explicitly confirmed by nonlocal measurements. Deviations from this behavior are found to be caused by thermally activated carriers, as indicated by an Arrhenius law temperature dependence. Using the deviations as a thermometer, we demonstrate an unexpected magnetocaloric effect and use it to reach near-perfect quantization by cooling the sample below the dilution refrigerator base temperature in a process approximating adiabatic demagnetization refrigeration.
\end{abstract}

The discovery of the quantum Hall effect (QHE) [1,2] led to a new understanding of electronic behavior in which topology plays a central role [3,4]. Initially, the critical experimental observation was the precise quantization of the Hall resistance to integer divisions of $h / e^{2}$, where $h$ is Planck's constant and $e$ is the electron charge. This quantization, immune to sample-specific disorder, now forms the basis for a metrological standard [5]. A complementary feature-zero longitudinal resistance, reflecting resistanceless transport along sample edges-could also have technological applications, were it not for the demanding environmental requirements for achieving the QHE: a large magnetic field to break time-reversal symmetry and, in most cases, cryogenic temperatures. Ideas for producing a similar phenomenology without an external magnetic field have long been considered [6], often involving the interplay of symmetry and topology in new material systems.

In the past decade, topological insulators (TIs) have emerged as a promising approach. In both two-dimensional [7-9] and three-dimensional [10-14] forms, conduction in TIs is restricted to topologically protected boundary states. In the 3D case, the presence of ferromagnetic exchange can break time-reversal symmetry, opening a gap in the otherwise Dirac-like surface states [15-17]. But topology adds a twist: even a uniformly magnetized sample will have, relative to the normal vector of the surface, a domain boundary where the magnetization switches from inward to outward. Along this line the gap should close, restoring conduction [16]. In a thin film geometry in which the easy axis of the magnetism is out of the plane, confinement along the sample side wall should ensure conduction is one dimensional while the surface gradient of the magnetism restricts it to only one direction, leading to ballistic, chiral transport. In a Hall bar geometry, this would be observed as the quantum anomalous Hall effect (QAHE), with a zero longitudinal resistance and a transverse resistance quantized to $h / n e^{2}$, where $n$ is typically \pm 1 but can in principle be a higher integer given sufficiently strong exchange [18].

Experimental realization of the QAHE has been swift. Doping films of the ternary TI family $(\mathrm{Bi}, \mathrm{Sb})_{2} \mathrm{Te}_{3}$ with $\mathrm{Mn}$ or $\mathrm{Cr}$ was found to produce robust out-of-plane ferromagnetism and a large anomalous Hall effect in transport [19-21]. Further growth optimization and chemical potential manipulation led to the recent achievement of the full quantized effect [22-24], albeit at dilution refrigerator temperatures. In two cases [22,24], an applied magnetic field was necessary to decrease the longitudinal resistivity, presumably to eliminate other conduction channels. The possibilities for these channels include nonchiral edge modes [25], variable-range hopping, or band transport of thermally populated 2D surface or 3D bulk carriers. In contrast, the device measured in Kou et al. [23] demonstrates its lowest longitudinal resistance near zero applied field.

In this Letter, we study the QAHE in this regime using material from the same growth, demonstrating the hallmarks of the effect: a vanishing longitudinal resistance, a precisely quantized Hall resistance that switches sign with magnetization, and direct confirmation of edge transport, all in the absence of an applied field. Where deviations from this ideal behavior occur, we attribute them to thermally activated carriers whose presence can be fortuitously controlled by demagnetization cooling of some other magnetic system in the sample. The material is ten quintuple layers of $\left(\mathrm{Cr}_{0.12} \mathrm{Bi}_{0.26} \mathrm{Sb}_{0.62}\right)_{2} \mathrm{Te}_{3}$ grown via molecular beam epitaxy and capped with alumina on a semi-insulating GaAs substrate. To avoid possible doping of the film through lithographic processing, following Ref. [22] we use a sharp metal 



FIG. 1 (color online). Device demonstrating quantum anomalous Hall effect. (a) Photograph of 10-nm-thick film of $\left(\mathrm{Cr}_{0.12} \mathrm{Bi}_{0.26} \mathrm{Sb}_{0.62}\right)_{2} \mathrm{Te}_{3}$ on a GaAs substrate, scratched by hand into a Hall bar shape, with indium metal Ohmic contacts. Schematic measurement setup included. (b) Longitudinal resistivity $\rho_{x x}$ and transverse resistivity $\rho_{y x}$ of the device at base temperature as a function of the applied magnetic field $\mu_{0} H$ in each sweep direction, forming a ferromagnetic hysteresis loop. As the field approaches zero from either starting point, $\rho_{y x}$ reaches its quantized value $h / e^{2}$ and $\rho_{x x}$ approaches zero. (c) Nonlocal and two-terminal measurements verifying edgedominated transport. The insets show the measurements performed and chirality at each magnetization.

tip to scratch the film into a Hall bar shape, and form Ohmic contacts by placing indium metal onto each terminal. The region between the voltage leads is $1.1 \mathrm{~mm}$ long and $0.45 \mathrm{~mm}$ wide [Fig. 1(a)]. Four-terminal resistances are measured via standard lock-in amplifier techniques [26] with the sample in a dilution refrigerator with its mixing chamber cooled to $38 \mathrm{mK}$. We calibrate the aggregate amplifier gain of the setup using a conventional $\nu=1$ quantum Hall plateau on a separate high-mobility graphene sample [26].

At base temperature we reproduce the ferromagnetic hysteresis loop measured by the anomalous Hall effect in Kou et al. [23] [Fig. 1(b)]. The sign of the transverse (Hall) resistivity $\rho_{y x}$ reflects the device's magnetization direction $M_{z}$, which we can set to positive ("+1") or negative ("-1") by applying a field $\mu_{0} H$ with a magnitude greater than the $125 \mathrm{mT}$ coercive field. As we sweep $H$ toward zero, $\rho_{y x}$ reaches its quantized value $\pm h / e^{2} \approx \pm 25,813 \Omega$ while the longitudinal resistivity $\rho_{x x}$ decreases precipitously (as low as $15 \Omega$ ). After crossing zero field, $\rho_{x x}$ increases to a few kilohms before spiking higher at the coercive field as $\rho_{y x}$ changes sign. Both measurements settle toward their quantized values as $|H|$ increases, but only reach full quantization on the return arm of the hysteresis loop, again just before zero field.

Although the resistivity tensor takes on the expected values, the hysteresis loop does not directly verify that edge conduction dominates in this regime. Nonlocal measurement configurations, such as that shown schematically in Fig. 1(c), are one way to establish this [35]. In the limit of chiral, ballistic edge transport, the chemical potential along the chirality direction only changes at leads that act as current sources or drains, as prescribed by the LandauerBüttiker formalism [36] and demonstrated in the QHE [37] (though not explicitly, to date, in the QAHE). For example, while flowing current between adjacent contacts (labeled 1 and 6), the remaining four contacts should maintain the same voltage as either the current source or drain, depending on whether the QAHE chirality is clockwise or counterclockwise, respectively. In Fig. 1(c) we measure the voltage drop from a contact on the opposite side of the device (contact 3 ) to the drain (contact 6), and plot the resulting three-terminal resistance $R_{16,36}$ compared to the two-terminal value $R_{16,16}$. At negative magnetization (left panel), corresponding to clockwise equilibration, both quantities approach the ballistic value $h / e^{2}$, indicating that contact 3 is nearly equilibrated with the source. At the opposite magnetization, where voltages are propagated counterclockwise (right panel), $R_{16,16}$ approaches $h / e^{2}$ while $R_{16,36}$ approaches zero due to the equilibration of contact 3 with the drain. Near zero field, the deviations from idealized behavior (in all cases under $200 \Omega$ ) likely reflect contact resistances and possibly the presence of extra dissipative helical edge modes [25]. Using one of the remaining contacts in the role of contact 3 results in the same behavior [26].

Returning to four-terminal measurements, we find that the best quantization can be obtained by maintaining the film's magnetization (i.e., keeping $\left|\mu_{0} H\right|$ smaller than the coercive field) but following the "hysteresis loops" shown in Fig. 2(a). Starting from any combination of magnetization and field polarity, sweeping $H$ toward zero suppresses the longitudinal conductivity $\sigma_{x x}=\rho_{x x} /\left(\rho_{x x}^{2}+\rho_{y x}^{2}\right)$ to as low as $0.0003 e^{2} / h$ while the transverse conductivity $\sigma_{x y}=\rho_{y x} /\left(\rho_{x x}^{2}+\rho_{y x}^{2}\right)$ remains quantized to within $0.01 \%$ of $e^{2} / h$. Passing through zero and then increasing $|H|$ destroys this quantization, which we can recover by waiting at constant field for $80 \mathrm{~min}$ and then sweeping back toward zero.

These reported conductivity values have undergone one correction for imperfect device geometry. Uneven spacing between the voltage probes of the Hall bar can add a small component of $\rho_{x x}$ to the measured value of $\rho_{y x}$. (In nonmagnetic samples, this is conventionally corrected by antisymmetrizing $\rho_{y x}$ about zero field.) In a parametric plot of 

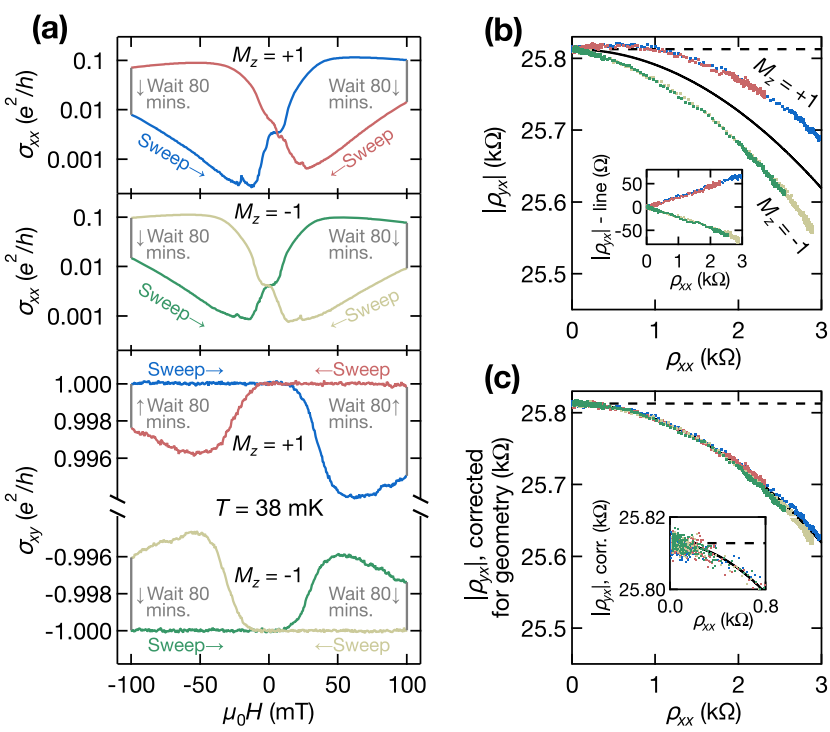

FIG. 2 (color online). Precise quantization near zero applied field. (a) Longitudinal and transverse conductivities in hysteresis loops over field ranges smaller than the coercive field so as to maintain the starting magnetization $M_{z}$. When approaching zero field from either starting point, $\sigma_{x y}=e^{2} / h$ to $0.01 \%$ precision while $\sigma_{x x}$ reaches as low as $0.0002 e^{2} / h$. (b),(c) Resistivities measured during the hysteresis loops, plotted parametrically, both before (b) and after (c) performing a correction for geometry. The inset of (b) shows the linear deviation of the two magnetization branches from a parabolic line, resulting from uneven spacing of the leads. The inset of (c) shows a close-up of the corrected resistivity data, with $\rho_{y x}$ quantized to $h / e^{2}$ within $3 \Omega$ whenever $\rho_{x x}<200 \Omega$.

the resistivity data from the same hysteresis loops [Fig. 2(b)], with $\left|\rho_{y x}\right|$ along the $y$ axis and $\rho_{x x}$ along the $x$ axis, we observe an asymmetry between the two magnetizations. Each branch deviates from a parabolic arc (the expected leading order contribution), with the size of the deviation explicitly verified to grow linearly with $\rho_{x x}$ with a coefficient of 2\% [Fig. 2(b), inset]. By taking field sweeps at $T=40 \mathrm{~K}$, above the film's Curie temperature [23], we obtain an independent but matching measure of this geometric mixing coefficient, which is also verified by a numerical Poisson simulation of current flow [26]. After removing the $\rho_{x x}$ component from $\rho_{y x}$ the data nearly collapse onto a single curve [Fig. 2(c)]. In the vicinity of vanishing $\rho_{x x}$, the parametric plot after this correction demonstrates quantization of $\rho_{y x}$ to within $\pm 3 \Omega$ [Fig. 2(c), inset].

The system stays on this curve in resistivity space, even during the wait times when $\rho_{x x}$ falls, suggesting that the position along the arc is determined by some parameter other than the magnetization or applied field. The temperature is an obvious possibility. We extend the relationship by warming the sample above $100 \mathrm{mK}$, inducing a large longitudinal conductivity that becomes comparable to $e^{2} / h$ by $750 \mathrm{mK}$ (Fig. 3). The trajectory of the QAHE in conductivity space during this process has been studied previously, and found to obey the same symmetry laws and

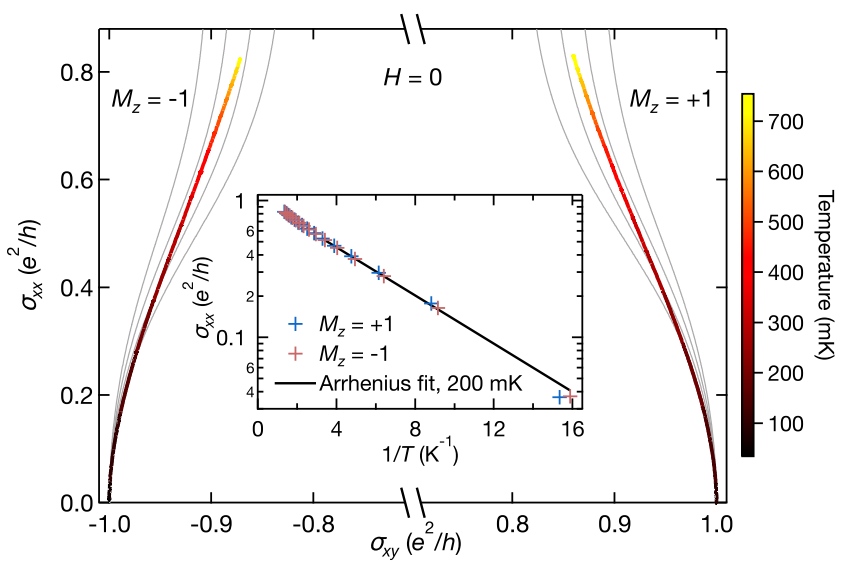

FIG. 3 (color online). Temperature dependence implying possible thermal activation. Parametric plot of $\sigma_{x x}$ versus $\sigma_{y x}$ at each magnetization while increasing temperature, with calculated renormalization group flow lines shown in gray. Inset: an Arrhenius plot of $\sigma_{x x}$ as a function of inverse temperature, showing roughly exponential dependence. The implied energy scale of the fit is $17 \mu \mathrm{eV}$, or $200 \mathrm{mK}$.

renormalization group properties as the QHE [24]. We find reasonable qualitative agreement with calculated [38] renormalization group flow lines (Fig. 3, gray lines).

To obtain a functional form of the temperature dependence, we plot $\sigma_{x x}$ versus reciprocal temperature (Fig. 3, inset). Such plots, useful for identifying conduction that is thermally activated over an energy barrier, are commonly used in quantum Hall systems to extract gap sizes [2]. Here, an Arrhenius (exponential) fit holds down to $60 \mathrm{mK}$, suggesting that the nonzero $\sigma_{x x}$ represents carriers thermally activated into a surface band. We emphasize that this is not a direct measurement of the size of the exchange gap: with no gate electrode, the chemical potential cannot be tuned to the middle of the surface gap. Furthermore, the fit continues to work well above the extracted energy scale, where describing thermal activation requires use of the Fermi-Dirac distribution as well as knowledge of the system's density of states and mobility as a function of energy [26]. Still, the data show some form of exponential activation suggesting that the nearest band edge is $17 \mu \mathrm{eV}$ away, as indicated by a $200 \mathrm{mK}$ characteristic temperature in the Arrhenius fit.

At the lowest temperatures, a sample's electron temperature can diverge from the cryostat's base temperature (here, that of the mixing chamber plate). In fact, the wide variation of $\sigma_{x x}$ in Fig. 2(a) indicates that the temperature may be changing substantially during a hysteresis loop: decreasing as the applied field is swept toward zero, and increasing once the field changes sign and is swept away from zero. We hypothesize that this magnetocaloric effect arises from the demagnetization and remagnetization of some spin system in thermal contact with the sample's electrons. The entropy of these spins can have a large dependence on the magnetic field: if the field's magnitude is decreased while the spins are held at constant temperature, then entropy increases as they depolarize. If, instead, 
(a)

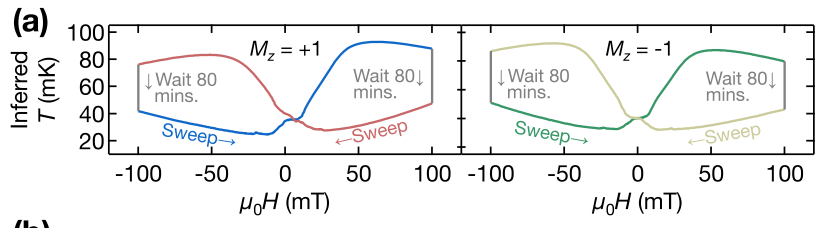

(b)
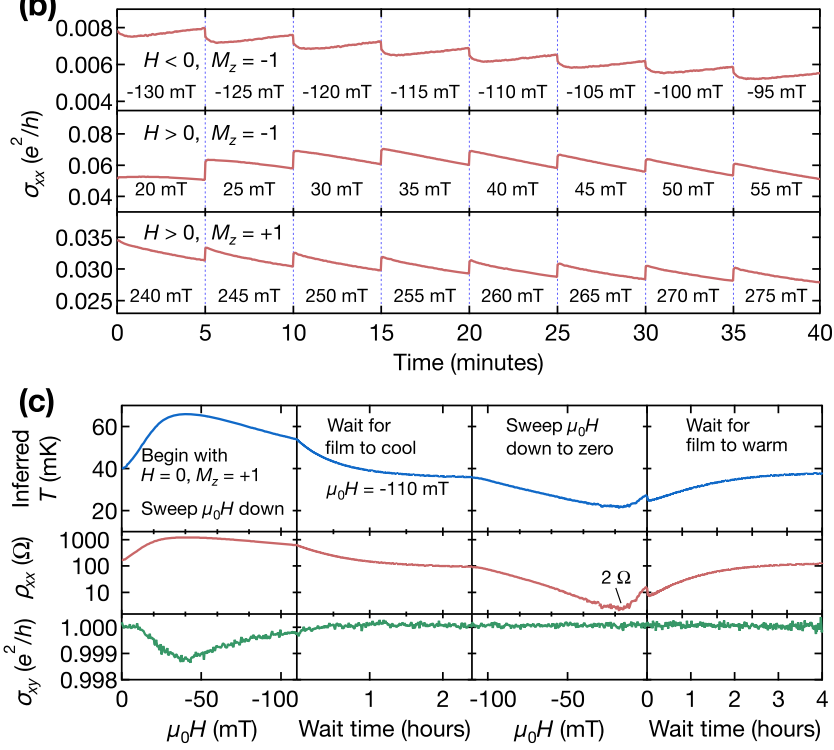

FIG. 4 (color online). Evidence for demagnetization cooling. (a) Inferred temperature of the TI film, extrapolated using the temperature fit in Fig. 3, during the hysteresis loop in Fig. 2(a). After a long wait, the temperature approaches the fridge thermometer reading $\sim 40 \mathrm{mK}$, whereas sweeping $|H|$ down drives the temperature below $30 \mathrm{mK}$ and sweeping $|H|$ up drives it above $90 \mathrm{mK}$. (b) Effect of upward field sweep on $\sigma_{x x}$ in three field and polarity regions, consistent with the demagnetization hypothesis. When $H<0$ (decreasing $|H|), \sigma_{x x}$ decreases when the field changes and creeps back up during a 5-min wait. When $H>0$ (increasing $|H|$ ), the opposite occurs regardless of magnetization. (c) A rudimentary demagnetization cycle, involving a slow field sweep to magnetize and a fast field sweep to zero to perform demagnetization cooling. Plotted during this cycle are the inferred temperature, $\rho_{x x}$ (now dropping below $2 \Omega$ ), and $\sigma_{x y}$, which remains within $0.01 \%$ of $e^{2} / h$ during the last three stages.

the combined system of electrons and spins is relatively isolated so that total entropy is maintained, its temperature will decrease as the field approaches zero [39]. (Conversely, increasing the field magnitude will lead to sample heating.) This is the principle behind adiabatic demagnetization refrigeration.

To check the reasonability of this hypothesis, we extrapolate the fit in Fig. 3 down to lower temperatures and apply it to the conductance data in Fig. 2(a) to obtain the approximate sample temperature during a hysteresis loop. The result [Fig. 4(a)] implies that the electron temperature after an 80-min wait at a constant field approaches the mixing chamber thermometer reading $\sim 40 \mathrm{mK}$. In downward field sweeps the sample drops below $30 \mathrm{mK}$, substantially below the refrigerator temperature, and in upward sweeps it exceeds $90 \mathrm{mK}$. (There are also smaller features, presumably heating events, around $\left|\mu_{0} H\right|=0$ and $20 \mathrm{mT}$ with unknown origins [26].)

We also test that the sample reequilibrates with the cryostat between field steps. While sweeping $\mu_{0} H$ from negative to positive, we stop for 5 min every $5 \mathrm{mT}$ to allow for partial equilibration. In a field region where $H$ is negative and its magnitude is decreasing [Fig. 4(b), top panel], $\sigma_{x x}$ decreases during the field step and then creeps back up during the wait time, demonstrating that the field step has cooled the sample below the cryostat temperature. At positive $H$ the opposite occurs [Fig. 4(b), middle panel] due to the now-warming effect of the field step. The pattern does not change after passing the coercive field and switching the film's magnetization [Fig. 4(b), bottom panel], indicating that only the polarity of the applied field matters, which in turn suggests that the system responsible for these temperature variations is distinct from the ferromagnetism measured in transport.

Although the exact identity of the system responsible for this magnetocaloric effect is otherwise unclear, a naive model of a paramagnet exchanging heat with the electrons qualitatively fits the shape of the temperature variation during demagnetization and suggests a Landé $g$ factor of $\sim 0.15$ [26]. We further believe it to be specific to and collocated with the TI sample: the refrigerator's thermometry shows minimal change during the hysteresis loop and, if we pause the loop during demagnetization, $\sigma_{x x}$ can remain below its equilibrium value for hours. Both details suggest that the TI surface and magnetic system are nearly thermally isolated from the cryostat when driven to their lowest temperatures. Further speculation on the origin of the effect, which has not been observed in materials from other sources, can be found in the Supplemental Material [26].

If we wish to minimize $\rho_{x x}$ or improve the quantization of $\sigma_{x y}$, an analogy to the traditional adiabatic demagnetization cycle is useful. A slow increase in the field's magnitude followed by a long wait, allowing maximal thermal equilibration along the way, approximates an isothermal magnetization step. Then, a fast decrease in the field magnitude (though not so fast that the heat load from the magnet sweep becomes relevant), produces adiabatic demagnetization. We plot $\rho_{x x}, \sigma_{x y}$, and the inferred temperature during this process in Fig. 4(c). Indeed, we drive $\rho_{x x}$ to as low as $2 \Omega$ $\left(\sigma_{x x}<0.0001 e^{2} / h\right)$ and the temperature to $25 \mathrm{mK}$, with excellent quantization in $\sigma_{x y}$ along the way. We also include a long wait at the end, demonstrating the long time scale for reequilibration of $\rho_{x x}$. Modifying this process to end at a small nonzero applied field, we can even drive $\rho_{x x}$ below $1 \Omega$ [26]. In future studies, adding a gate electrode to optimize the position of the chemical potential in the gap may yield a completely vanishing longitudinal resistance.

Sample preparation, measurements, and analysis were supported by the U.S. Department of Energy, Office of Science, Basic Energy Sciences, under Award No. 19-7503. Materials growth, surface characterization, preliminary electrical characterization, and electronic instrumentation were 
supported by the DARPA MESO program under Contracts No. N66001-12-1-4034 and No. N66001-11-1-4105. Infrastructure and cryostat support were funded in part by the Gordon and Betty Moore Foundation through Grant No. GBMF3429 to D. G.-G. The authors acknowledge Stefan Grauer and Charles Gould for proposing the nonlocal measurements and for insightful technical discussions, Jing Wang, Biao Lian, and Xiaoliang Qi for theoretical comments, Yang Feng and $\mathrm{Ke} \mathrm{He}$ for stimulating discussions about material properties, Jingsong Zhang for help with defining Hall bars, and Lucas Peeters for help with device measurement. K. L.W. acknowledges the support of the Raytheon endorsement. A. J. B. acknowledges support from a Benchmark Stanford Graduate Fellowship, E. J. F. acknowledges support from a DOE Office of Science Graduate Fellowship, and X. K. acknowledges partial support from a Qualcomm Innovation Fellowship.

Note added.-Recently, we became aware of work by Chang et al. that reports a similar degree of quantization in V-doped BiSbTe $_{2}[40]$.

*To whom all correspondence should be addressed. goldhaber-gordon@stanford.edu

[1] K. v. Klitzing, G. Dorda, and M. Pepper, Phys. Rev. Lett. 45, 494 (1980).

[2] D. C. Tsui, H. L. Stormer, and A. C. Gossard, Phys. Rev. Lett. 48, 1559 (1982).

[3] R. B. Laughlin, Phys. Rev. B 23, 5632 (1981).

[4] D. J. Thouless, M. Kohmoto, M. P. Nightingale, and M. den Nijs, Phys. Rev. Lett. 49, 405 (1982).

[5] K. von Klitzing, Phil. Trans. R. Soc. A 363, 2203 (2005).

[6] F. D. M. Haldane, Phys. Rev. Lett. 61, 2015 (1988).

[7] C. L. Kane and E. J. Mele, Phys. Rev. Lett. 95, 146802 (2005)

[8] B. A. Bernevig, T. L. Hughes, and S.-C. Zhang, Science 314, 1757 (2006).

[9] M. König, S. Wiedmann, C. Brüne, A. Roth, H. Buhmann, L. W. Molenkamp, X.-L. Qi, and S.-C. Zhang, Science 318, 766 (2007).

[10] L. Fu, C. L. Kane, and E. J. Mele, Phys. Rev. Lett. 98, 106803 (2007).

[11] X.-L. Qi, T. L. Hughes, and S.-C. Zhang, Phys. Rev. B 78, 195424 (2008).

[12] D. Hsieh, Y. Xia, D. Qian, L. Wray, J. H. Dil, F. Meier, J. Osterwalder, L. Patthey, J. G. Checkelsky, N. P. Ong, A. V. Fedorov, H. Lin, A. Bansil, D. Grauer, Y. S. Hor, R. J. Cava, and M.Z. Hasan, Nature (London) 460, 1101 (2009).

[13] M. Z. Hasan and C. L. Kane, Rev. Mod. Phys. 82, 3045 (2010).

[14] X.-L. Qi and S.-C. Zhang, Rev. Mod. Phys. 83, 1057 (2011).

[15] R. Yu, W. Zhang, H.-J. Zhang, S.-C. Zhang, X. Dai, and Z. Fang, Science 329, 61 (2010).

[16] K. Nomura and N. Nagaosa, Phys. Rev. Lett. 106, 166802 (2011).

[17] Y. L. Chen, J.-H. Chu, J. G. Analytis, Z. K. Liu, K. Igarashi, H.-H. Kuo, X. L. Qi, S. K. Mo, R. G. Moore, D. H. Lu,
M. Hashimoto, T. Sasagawa, S. C. Zhang, I. R. Fisher, Z. Hussain, and Z. X. Shen, Science 329, 659 (2010).

[18] J. Wang, B. Lian, H. Zhang, Y. Xu, and S.-C. Zhang, Phys. Rev. Lett. 111, 136801 (2013).

[19] J. G. Checkelsky, J. Ye, Y. Onose, Y. Iwasa, and Y. Tokura, Nat. Phys. 8, 729 (2012).

[20] C.-Z. Chang, J. Zhang, M. Liu, Z. Zhang, X. Feng, K. Li, L.-L. Wang, X. Chen, X. Dai, Z. Fang, X.-L. Qi, S.-C. Zhang, Y. Wang, K. He, X.-C. Ma, and Q.-K. Xue, Adv. Mater. 25, 1065 (2013).

[21] X. Kou, M. Lang, Y. Fan, Y. Jiang, T. Nie, J. Zhang, W. Jiang, Y. Wang, Y. Yao, L. He, and K. L. Wang, ACS Nano 7, 9205 (2013).

[22] C.-Z. Chang et al., Science 340, 167 (2013).

[23] X. Kou, S.-T. Guo, Y. Fan, L. Pan, M. Lang, Y. Jiang, Q. Shao, T. Nie, K. Murata, J. Tang, Y. Wang, L. He, T.-K. Lee, W.-L. Lee, and K. L. Wang, Phys. Rev. Lett. 113, 137201 (2014).

[24] J. G. Checkelsky, R. Yoshimi, A. Tsukazaki, K. S. Takahashi, Y. Kozuka, J. Falson, M. Kawasaki, and Y. Tokura, Nat. Phys. 10, 731 (2014).

[25] J. Wang, B. Lian, H. Zhang, and S.-C. Zhang, Phys. Rev. Lett. 111, 086803 (2013).

[26] See Supplemental Material http://link.aps.org/supplemental/ 10.1103/PhysRevLett.114.187201, for further information about measurement setup and calibration, more nonlocal measurements, analysis of demagnetization, and additional supporting data, which includes Refs. [27-34].

[27] L. He, X. Kou, M. Lang, E. S. Choi, Y. Jiang, T. Nie, W. Jiang, Y. Fan, Y. Wang, F. Xiu, and K. L. Wang, Sci. Rep. 3, 3406 (2013)

[28] M. Lang, L. He, F. Xiu, X. Yu, J. Tang, Y. Wang, X. Kou, W. Jiang, A. V. Fedorov, and K. L. Wang, ACS Nano 6, 295 (2012).

[29] F. P. Milliken, J. R. Rozen, G. A. Keefe, and R. H. Koch, Rev. Sci. Instrum. 78, 024701 (2007).

[30] F. Fischer and M. Grayson, J. Appl. Phys. 98, 013710 (2005).

[31] P. L. Gammel, D. J. Bishop, J. P. Eisenstein, J. H. English, A. C. Gossard, R. Ruel, and H. L. Stormer, Phys. Rev. B 38, 10128 (1988).

[32] C.-Z. Chang, P. Tang, Y.-L. Wang, X. Feng, K. Li, Z. Zhang, Y. Wang, L.-L. Wang, X. Chen, C. Liu, W. Duan, K. He, X.-C. Ma, and Q.-K. Xue, Phys. Rev. Lett. 112, 056801 (2014).

[33] K. Elliott, R. T. Chen, S. G. Greenbaum, and R. J. Wagner, Appl. Phys. Lett. 44, 907 (1984).

[34] J. S. Blakemore, J. Appl. Phys. 53, R123 (1982).

[35] A. Roth, C. Brüne, H. Buhmann, L. W. Molenkamp, J. Maciejko, X.-L. Qi, and S.-C.Zhang, Science 325, 294 (2009).

[36] M. Büttiker, Phys. Rev. B 38, 9375 (1988).

[37] C. Beenakker and H. van Houten, in Semiconductor Heterostructures and Nanostructures, Solid State Physics, Vol. 44, edited by H. Ehrenreich and D. Turnbull (Academic Press, New York, 1991) pp. 1-228.

[38] B. P. Dolan, Nucl. Phys. B554, 487 (1999).

[39] V. K. Pecharsky, K. A. Gschneidner, A. O. Pecharsky, and A. M. Tishin, Phys. Rev. B 64, 144406 (2001).

[40] C.-Z. Chang, W. Zhao, D. Y. Kim, H. Zhang, B. A. Assaf, D. Heiman, S.-C. Zhang, C. Liu, M. H. W. Chan, and J. S. Moodera, arXiv:1412.3758 [Nature (London) (to be published). 